\title{
User Identity Protection in Automatic Emotion Recognition through Disguised Speech
}

\author{
Fasih Haider *(D), Pierre Albert (D) and Saturnino Luz \\ Usher Institute, Edinburgh Medical School, The University of Edinburgh, Edinburgh EH16 4UX, UK; \\ pierre.albert@ed.ac.uk (P.A.); S.Luz@ed.ac.uk (S.L.) \\ * Correspondence: Fasih.Haider@ed.ac.uk
}

Citation: Haider, F.; Albert, P.; Luz, S. User Identity Protection in Automatic Emotion Recognition through Disguised Speech. AI 2021, 2, 636-649. https://doi.org/ 10.3390/ai2040038

Academic Editor: Friedhelm Schwenker and Mariofanna Milanova

Received: 19 October 2021

Accepted: 22 November 2021

Published: 25 November 2021

Publisher's Note: MDPI stays neutral with regard to jurisdictional claims in published maps and institutional affiliations.

Copyright: (c) 2021 by the authors. Licensee MDPI, Basel, Switzerland. This article is an open access article distributed under the terms and conditions of the Creative Commons Attribution (CC BY) license (https:// creativecommons.org/licenses/by/ $4.0 /)$.

\begin{abstract}
Ambient Assisted Living (AAL) technologies are being developed which could assist elderly people to live healthy and active lives. These technologies have been used to monitor people's daily exercises, consumption of calories and sleep patterns, and to provide coaching interventions to foster positive behaviour. Speech and audio processing can be used to complement such AAL technologies to inform interventions for healthy ageing by analyzing speech data captured in the user's home. However, collection of data in home settings presents challenges. One of the most pressing challenges concerns how to manage privacy and data protection. To address this issue, we proposed a low cost system for recording disguised speech signals which can protect user identity by using pitch shifting. The disguised speech so recorded can then be used for training machine learning models for affective behaviour monitoring. Affective behaviour could provide an indicator of the onset of mental health issues such as depression and cognitive impairment, and help develop clinical tools for automatically detecting and monitoring disease progression. In this article, acoustic features extracted from the non-disguised and disguised speech are evaluated in an affect recognition task using six different machine learning classification methods. The results of transfer learning from non-disguised to disguised speech are also demonstrated. We have identified sets of acoustic features which are not affected by the pitch shifting algorithm and also evaluated them in affect recognition. We found that, while the non-disguised speech signal gives the best Unweighted Average Recall (UAR) of $80.01 \%$, the disguised speech signal only causes a slight degradation of performance, reaching $76.29 \%$. The transfer learning from non-disguised to disguised speech results in a reduction of UAR (65.13\%). However, feature selection improves the UAR (68.32\%). This approach forms part of a large project which includes health and wellbeing monitoring and coaching.
\end{abstract}

Keywords: privacy preservation; affect recognition; health technologies; emotion recognition; Ambient Assisted Living; social signal processing

\section{Introduction}

Health and wellbeing monitoring using Ambient Assisted Living (AAL) technologies involves developing systems for automatically detecting and tracking a number of events that might require attention or coaching. In the SAAM project [1], we are employing AAL technologies to analyse activities and health status of older people living on their own or in assisted care settings, and to provide them with personalised multimodal coaching. Such activities and status include mobility, sleep, social activity, air quality, cardiovascular health, diet [2], emotions [3] and cognitive status [4]. While most of these signals are tracked through specialized hardware, audio and speech are ubiquitous sources of data which could also be explored in these contexts. Speech quality and activity, in particular, closely reflect health and wellbeing. We have explored the potential of speech analysis for automatically recognizing emotions [3], cognitive difficulties [4] and eatingrelated events [2] in the SAAM AAL environment [5]. AAL technologies and coaching systems such as SAAM, which focus on monitoring of everyday activities, can benefit from 
recognition of these audio events in characterizing contextual information against which other monitoring signals can be interpreted. However, user privacy remains one of the major challenges in collecting audio data in home environments for the development of health monitoring technology.

\subsection{Mental Health and Affective Speech}

The literature suggests that older people with cognitive impairment have difficulty accessing semantic information when they intend to do so [6]. Since successful communication is essential for meaningful social interaction, this takes a toll on patients' and their carers' wellbeing. This has an impact on the emotional life of these people. Speech monitoring for mood and cognitive changes may help inform interventions targeted at alleviating such impacts.

In addition to their role in cognition [7], the expression of emotions and their recognition are key aspects of communication [8]. Emotional information can be conveyed in different ways, from explicit facial and verbal expression (e.g., smile, pout, happy statement) to more subtle non-verbal cues, such as intonation, modulation of vocal pitch and loudness of emotional expression. These non-verbal cues are generally referred to as emotional prosody.

In a previous study [9], we found that there are differences in automatically inferred affective behaviours regarding expressions of sadness,anger and disgust among people with and without cognitive impairment (Alzheimer's Disease, AD). Although these results need further study, they suggest, in agreement with the incipient literature on this topic that speakers with AD exhibit a deficit in the expression of those emotions, reflected on voice volume, speech rate and pitch. The proposed Affective Behaviour Representation (ABR) and emotion classification scores are able to predict cognitive deficit in such situations with an accuracy of $63.42 \%$. However, in that study, there was a mismatch between the dataset used to generate the features for recognition (emoDB [10]) and the data on which these features were used (Pitt Corpus [11]). Thus, prediction accuracy is likely to have been hindered by the facts that (1) the Pitt Corpus was not explicitly designed to elicit emotions, (2) that the two datasets were recorded under different acoustic conditions, (3) that the speakers were selected from different demographics, and (4) that they are in different languages [9].

\subsection{Privacy-Concerns Related to Speech}

Privacy concerns constitute a major obstacle in developing and deploying digital technologies for monitoring cognitive health. Individual and societal concerns about privacy and data security have been translated in regulations. In the European Union, the GDPR [12] has set new standards for the collection and management of personal information. Speech data are classified as personal data (as defined in Art. 4(14) of the GDPR and Article 3(13) Directive 2016/680): it can be used to identify age, gender, subject identity and health status [13]. Sensitive data also encompass additional data such as content-free features which could potentially be used for the identification of a person. The potential of such features as biometric markers further widens the importance of their protection. Concern about privacy is shared by users, who are reluctant to consent to being constantly recorded at their homes and/or while speaking through phones or computers. The balance between the benefits from an analysis of spoken interaction is often offset by the associated threat to privacy.

Ethical requirements for health-studies have reflected these changes in regulation. They have raised awareness on the need for careful risk analysis for studies involving the collection and use of speech-related data. In the context of AAL and in-situ studies, speech analysis usually requires sending data over networks with different levels of security and associated risks, setting the additional possibility of a data breach if intercepted and compromised. While the security of the network can be improved by reducing the transit 
and exposure of sensitive data through a local pre-processing [14,15], the risk posed by the presence of sensitive data remains.

A solution to these problems is to obfuscate the identity of a person while the data are collected, for instance through changing the pitch of their speech [16]. However, changing the signal can also degrade its analysis: pitch shifting disturbs the acoustic patterns of speech which could be indicative of cognitive impairment.

Hence, developing a digital technology using acoustic information should take these issues into account. In this study, we also propose a framework using feature engineering to address the disturbance of acoustic features caused by pith alteration for affect recognition as described in Section 4.4.

\subsection{Speech Disguising}

Speech Disguising is a way to alter speech to hide someone's identity [16]. Zheng et al. [17] subjectively analyse the automatic speech disguise technologies i.e., pitch shifting, vocal tract length normalization (VTLN) and voice conversion (VC) using 30 trials. It is found that the speech disguise technologies greatly confuse the humans evaluators, with an equal error rate around random guess (i.e., $50.00 \%$ for pitch shifting, $46.67 \%$ for VTLN and $46.67 \%$ for VC).

\subsection{Contribution}

We have previously developed a low-cost system $[15,18]$ which records content-free, anonymised audio features for automatic analysis. In particular, we extract features such as the eGeMAPS set [19] which we have used to detect specific behaviours in the abovementioned applications [2-4]. However, one of the limitations was that the previous system [15] deletes the audio file after extracting the acoustic features from user's speech. It could work if the emotion is self-reported by user, and we do not have a plan to evaluate the new features (i.e., going to be proposed in future), but not for situations where other humans needs to annotate the audio files with emotions to generate data for machine learning model training. Thus, that preserving audio file is also important while preserving privacy. The disguised speech technologies could help us in preserving user's privacy to some extent, but a question arises: "is there any benefit of acoustic information in disguised speech for emotion recognition"? In this study, we extend our previous work and propose to collect the disguised speech by altering the pitch of the speech signal to protect the identity of a user for development and deployment of machine learning based application. For testing (i.e., deployment), this approach also guarantees the user's spoken content privacy in addition to identity protection. This is because the acoustic features are computed using different statistical functionals at the utterance level rather than at frame level, which makes it impossible to extract or re-build content information through, for instance, synthesis of speech from the extracted features or automatic transcription [20].

To the authors' best knowledge, this is the first study and evaluation of disguised speech for the development and deployment of affect recognition technologies based on acoustic features. Hence, the contributions of this article are as follows:

- Identification of acoustic features which are not affected by disguising speech;

- Evaluation of acoustic features extracted from the disguised speech for affect recognition, and comparison with features extracted from non-disguised speech;

- Demonstration of transfer-learning of acoustic features from non-disguised speech to disguised speech for affect recognition, and analysis of their generalisability.

\section{Materials and Methods}

This section describes the system and algorithms which have been used for proposing emotion recognition using disguised speech. 


\subsection{Emotion Recognition System}

This section describes hardware and software components of the system used to extract acoustic features and collect disguised speech. The collected disguised speech could be presented to human annotators (e.g., crowd-sourced annotation i.e., labelling stage) for annotation of emotions. The system's architecture is shown in Figure 1 where voice activity detection module detects audio segments based on the energy of audio signals. After that, we use pitch shifting algorithm [21] for speech disguising and saves the audio segments. Later, we extract acoustic features using openSMILE [19] and train machine learning models (i.e., Development module) for emotion recognition. At the end, we test the machine learning model (i.e., affective and emotional processing module).

\subsubsection{Hardware Components}

The hardware consists of a Matrix Creator board, constituted of a microphone array, an inertial measurement unit, and several other sensors, mounted on a Raspberry Pi $3 \mathrm{~B}+$, as shown in Figure 2. This setup is meant to be installed in a room where social activity and dialogue interaction occur frequently, such as a dining room or a sitting room.

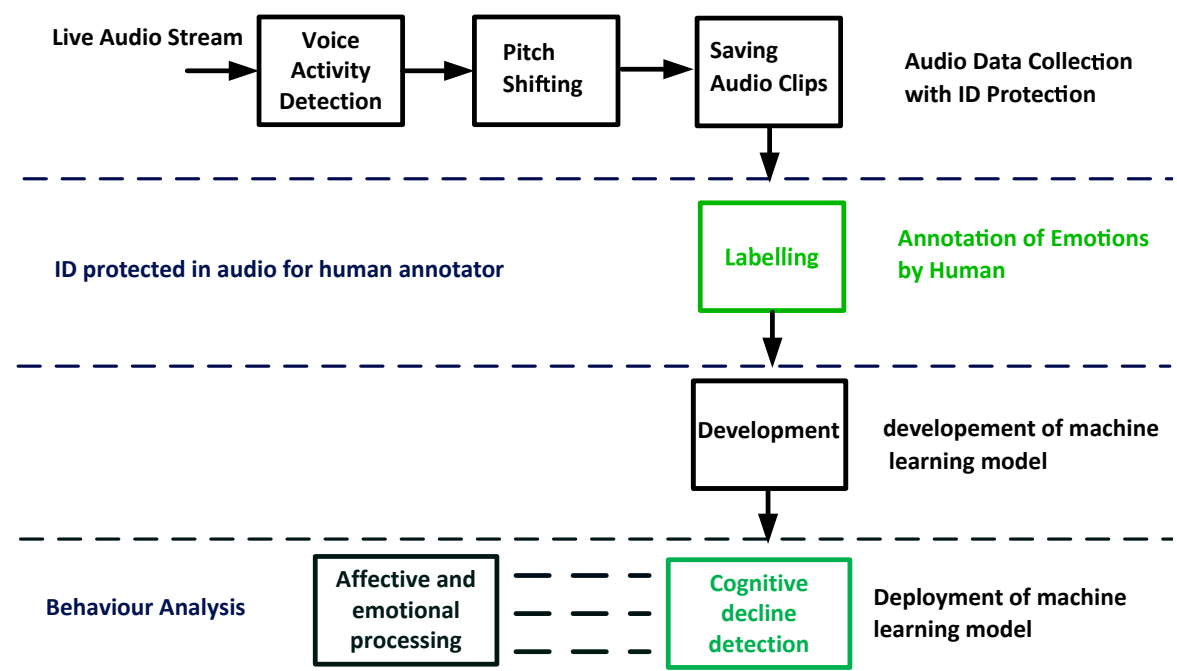

Figure 1. Proposed approach: the affective and emotional processing module will provide input to the cognitive decline recognition module. The 'labelling' and 'cognitive decline detection' are not part of this study. The pitch shifting parameters are only known to and set by the data collection technician and/or user. The Human annotator does not have that information.

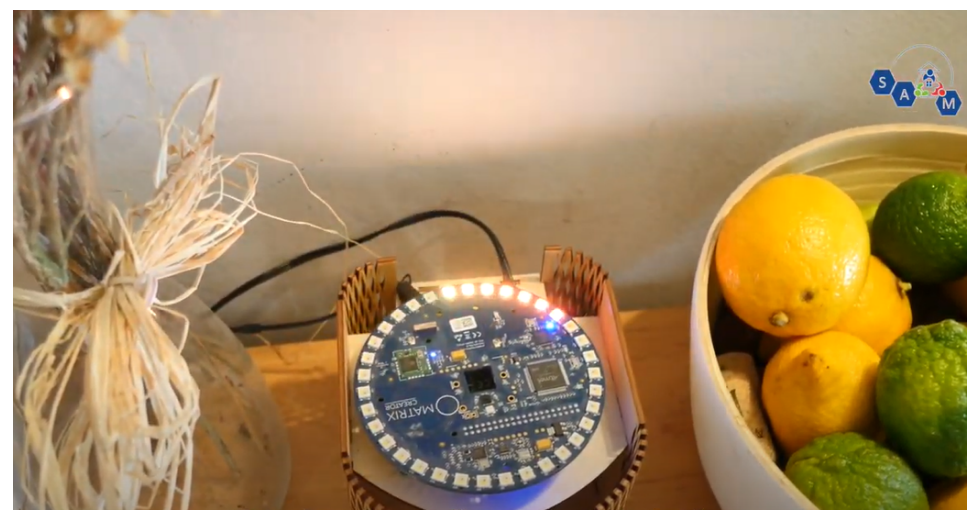

Figure 2. Matrix Creator and Raspberry Pi 3 B+.

\subsubsection{Software Components}

For voice activity detection, we employed the Auditok (https://pypi.org/project/ auditok/—accessed 21 November 2021) Python binding. Based on watchdog https://github. 
com/gorakhargosh/watchdog-accessed 21 November 2021 input, the OpenSMILE [22] toolkit processes the audio file of disguised speech and saves the speech features in the attribute-relation file format (ARFF). The extracted acoustic features are then processed by a machine learning model to identify the emotion(s).

\subsection{Data Sets}

The Berlin Database of Emotional Speech (EmoDB) corpus [10] is a data set commonly used in the automatic emotion recognition literature. It features 535 acted emotions in German ( 5 male and 5 females), based on utterances carrying no emotional bias. The corpus was recorded in a controlled environment resulting in high quality recordings. Actors were allowed to move freely around the microphones, which affected absolute signal intensity. In addition to the emotion, each recording was labelled with phonetic transcription using the SAMPA phonetic alphabet, emotional characteristics of the voice, segmentation of the syllables, and stress. The quality of the data set was evaluated by perception tests carried out by 20 human participants. In a first recognition test, subjects listened to a recording once before assigning one of the available categories, achieving an average recognition rate of $86 \%$. A second naturalness test was performed. Documents achieving a recognition rate lower than $80 \%$ or a naturalness rate lower than $60 \%$ were discarded from the main corpus, reducing the corpus to 535 recordings from the original 800 . The data sets are annotated for $6+1$ emotions: anger, disgust, fear, joy (happiness), sadness, and boredom + neutral.

\subsection{Identity Protection}

To disguise the identify of the subjects, we apply pitch shifting algorithm while maintaining the duration of speech signal using Praat [21]. The audio data with identity protection along with script for pitch shifting are made available through our git repository (https: / / git.ecdf.ed.ac.uk/fhaider/pitchshifting4affectrecogntion-accessed 21 November 2021 ). We have used a factor of 2 for pitch shifting with time step of $0.01 \mathrm{~s}$, minimum pitch of $75 \mathrm{~Hz}$, and maximum pitch of $600 \mathrm{~Hz}$. The pitch shifting parameters are only known to and set by the data collection technician and/or user. The Human annotator does not have that information. An example of non-disguised and disguised audio segment (i.e., spectrogram representation) is shown in Figures 3 and 4, respectively, where the duration of non-disguised and disguised speech is the same.

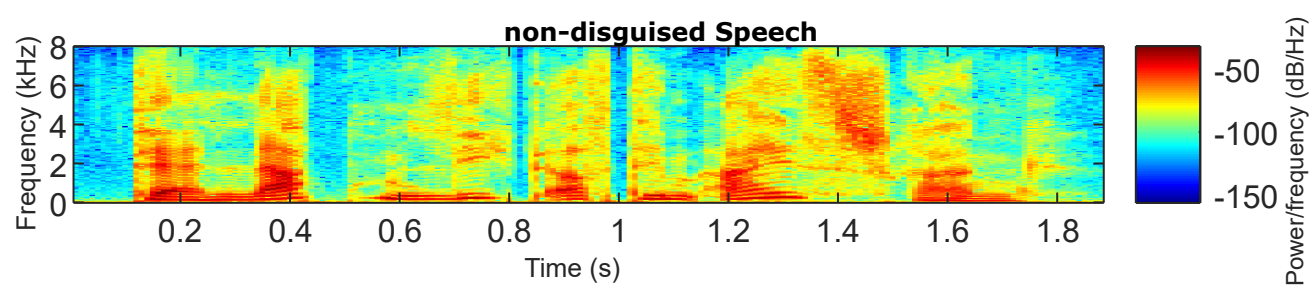

Figure 3. An example of a speech utterance's spectrogram from the EmoDB dataset of a male subject.

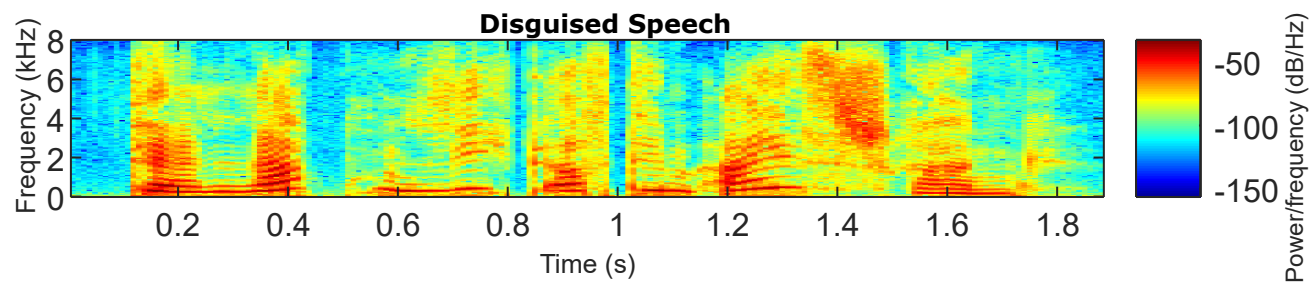

Figure 4. An example of a speech utterance's spectrogram from the EmoDB dataset of a male subject after applying pitch shifting algorithm for identity protection.

\subsection{Acoustic Features}

Acoustic feature extraction was performed on the non-disguised and disguised speech segments using the openSMILE v2.1 toolkit, which is a "source-available" software suite for automatic extraction of features from speech, widely used for emotion and affect 
recognition in speech [23]. The extracted features are also made available through gitrepository. The following is a brief description of the acoustic feature sets used in the experiments described in this paper:

\subsubsection{Emobase}

This feature set contains the mel-frequency cepstral coefficients (MFCC), voice quality, fundamental frequency (F0), F0 envelope, line spectral pairs (LSP) and intensity features with their first and second order derivatives. Several statistical functions are applied to these features, resulting in a total of 988 features for every speech segment [23].

\subsubsection{ComParE}

The ComParE 2013 [22] feature set includes energy, spectral, MFCC, and voicing related low-level descriptors (LLDs). LLDs include logarithmic harmonic-to-noise ratio, voice quality features, Viterbi smoothing for F0, spectral harmonicity and psychoacoustic spectral sharpness. Statistical functionals are also computed, bringing the total to 6373 features.

\subsection{3. eGeMAPS}

The eGeMAPS [19] feature set resulted from an attempt to reduce the somewhat unwieldy feature sets above to a reduced set of acoustic features based on their potential to detect physiological changes in voice production, as well as theoretical significance and proven usefulness in previous studies [19]. It contains the F0 semitone, loudness, spectral flux, MFCC, jitter, shimmer, F1, F2, F3, alpha ratio, Hammarberg index and slope V0 features, as well as their most common statistical functionals, for a total of 88 features per speech segment.

\subsection{Statistical Analysis}

To investigate the possible differences in acoustic characteristics between the nondisguised and disguised speech signals, we first performed a normality test using the one-sample Kolmogorov-Smirnov procedure. This test showed that the data (i.e., acoustic features) follow a normal distribution $(p<0.001)$. We then performed a $t$-test between the acoustic features extracted from the non-disguised speech signals and the acoustic features extracted from the disguised speech signal. We observed the following:

1. for the emobase feature set, there are 257 features out of 988 for which no statistically significant differences $(p>0.05)$ between the non-disguised and disguised speech signals were found. Parts of different functional of Mfcc, fftMag, ZCR, energy, loudness and intensity are not affected by the speech alteration.

2. For the ComParE feature set, we found that 2491 features out of 6373 show no statistically significant differences $(p>0.05)$ between non-disguised and disguised speech signals. Some mfcc, fftMag, audiospec, HNR, ZCR, energy, RASTA, jitter and shimmer functionals are not affected by the speech alteration procedure. The full lists of emobase and ComParE features tested are available through the above-mentioned git repository.

3. For the eGeMAPS feature set, we have noted that there are 24 features out of 88 which have no statistically significant differences $(p>0.05)$. The full list of those features is shown below:

- F0semitoneFrom $27.5 \mathrm{~Hz} \_s m a 3 n z \_p c t$ lrange $0-2$

- F0semitoneFrom27.5Hz_sma3nz_meanRisingSlope

- F0semitoneFrom27.5Hz_sma3nz_stddevRisingSlope

- F0semitoneFrom27.5Hz_sma3nz_stddevFallingSlope

- loudness_sma3_meanRisingSlope

- spectralFlux_sma3_stddevNorm

- mfcc1_sma3_stddevNorm

- mfcc2_sma3_stddevNorm

- mfcc3_sma3_stddevNorm 
- $\quad \log R e l F 0-H 1-H 2 \_s m a 3 n z \_s t d d e v N o r m$

- $\quad \log R e l F 0-H 1-A 3 \_s m a 3 n z \_s t d d e v N o r m$

- alphaRatioV_sma3nz_amean

- alphaRatioV_sma3nz_stddevNorm

- hammarbergIndexV_sma3nz_amean

- $\quad$ slopeV0 - 500_sma3nz_stddevNorm

- $\quad$ slopeV500 - 1500_sma3nz_stddevNorm

- $\quad$ spectralfluxV_sma3nz_stddevNorm

- $m f c c 1 V_{s} m a 3 n z \_s t d d e v N o r m$

- $\quad m f c c 2 V_{s} m a 3 n z \_s t d d e v N o r m$

- $m f c c 3 V_{s} m a 3 n z \_s t d d e v N o r m$

- $m f c c 4 V_{s} m a 3 n z \_s t d d e v N o r m$

- loudnessPeaksPerSec

- MeanUnvoicedSegmentLength

- StddevUnvoicedSegmentLength.

\subsection{Classification Methods}

The classification experiments were performed using six different methods, namely decision trees (DT, where the leaf size is optimized through a grid search within a range of 1 to 20), nearest neighbour (KNN, where K parameter is optimized through a grid search within a range of 1 to 10), linear discriminant analysis (LDA), random forest (RF, with 1500 trees, where leaf size is optimized through a grid search within a range of 1 to 20), Naive Bayes (NB, with kernel distribution assumption optimized through a grid search for kernel smoothing density estimate, Multinomial distribution, Multivariate multinomial distribution and Normal distribution) and support vector machines: SVM, with a linear kernel (optimized by trying different kernel function i.e., linear, Gaussian, RBF and polynomial) with box constraint optimized by trying a grid search between 0.1 to 1.0, and sequential minimal optimization solver (optimized by trying different solvers i.e., iterative single data algorithm, L1 soft-margin minimization by quadratic programming and sequential minimal optimization). The prior-probabilities of the classifiers are set according to the class distributions.

The classification methods are implemented in MATLAB (http:/ /uk.mathworks.com/ products/matlab / (accessed 21 November 2021)) using the statistics and machine-learning toolbox. The classifier hyper-parameters maximum ranges (such as $K=10$ ) are set through trial and error. A leave-one-subject-out (LOSO) cross-validation setting was adopted, where the training data do not contain any information of the validation subjects. To assess the classification results, we used the Unweighted Average Recall (UAR) instead of overall accuracy as the dataset is imbalanced. The Unweighted Average Recall is the arithmetic mean of recall for all seven classes.

\section{Experimentation}

This section describes the experiments and data partition to evaluate the proposed frameworks as shown in Figure 5.

\subsection{Experiment 1}

In this experiment, we extracted acoustic features over the non-disguised audio data. Later, we trained the machine learning models for classification purpose. The validation is performed in leave-one subject out cross-validation setting as shown in Figure 5a.

\subsection{Experiment 2}

In this experiment, we extracted acoustic features over the transformed audio data where we hid the identity of a subject using pitch shifting algorithm. Later, we trained the machine learning models for classification purpose. The validation is performed in leave-one subject out cross-validation setting as shown in Figure 5b. 


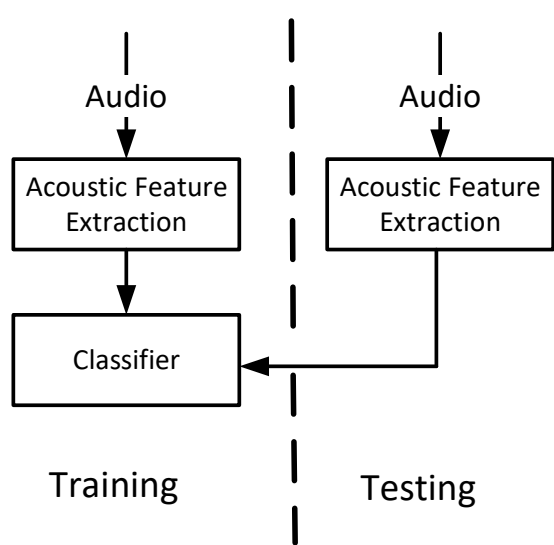

(a) Experiment 1

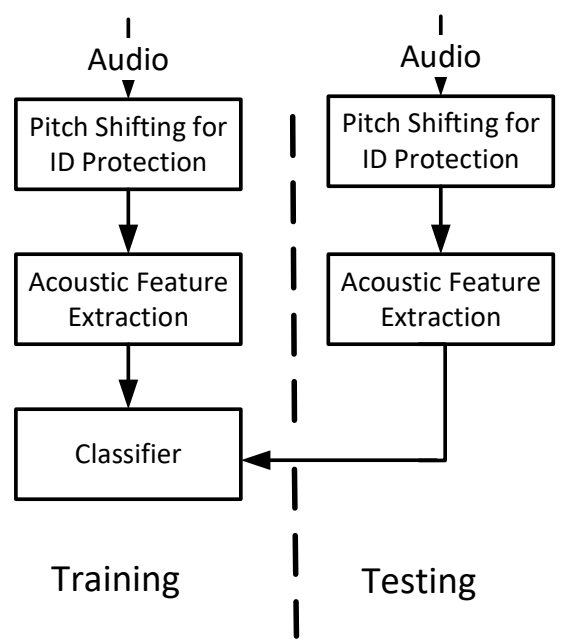

(b) Experiment 2

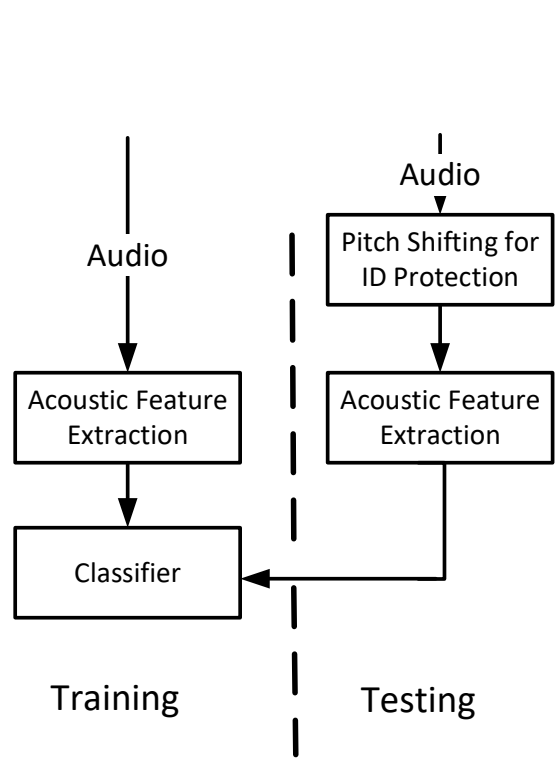

(c) Experiment 3

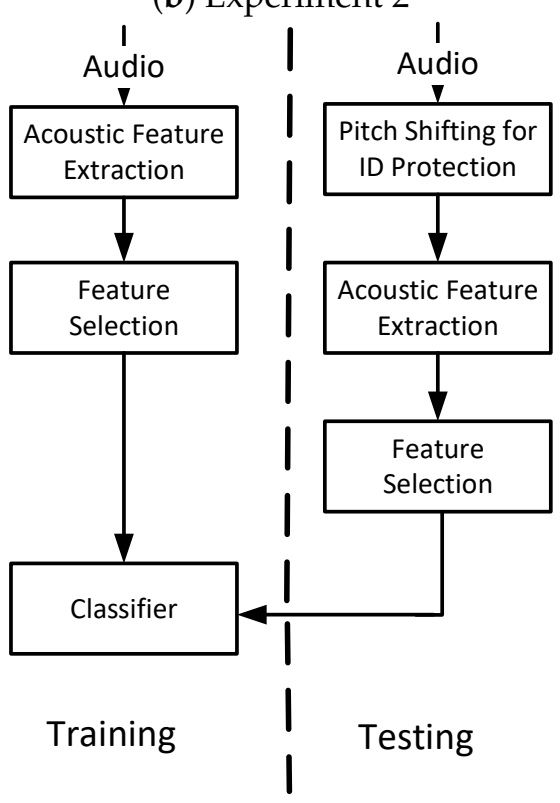

(d) Experiment 4

Figure 5. Affect recognition system: Machine learning model training and testing where testing is performed in leave one subject out cross-validation settings.

\subsection{Experiment 3}

In this experiment, we trained the machine learning models using non-disguised speech and the validation is performed using disguised speech in leave-one subject out cross-validation setting as shown in Figure 5c.

\subsection{Experiment 4}

This experiment uses the selected acoustic features as described in Section 2.5, we trained the machine learning models using non-disguised speech and the validation is performed using disguised speech in leave-one subject out cross-validation setting as shown in Figure 5d.

\section{Results}

This section reports the results for the four experiments.

\subsection{Experiment 1}

The UAR for all feature sets and classification methods is shown in Table 1. These results indicate that the ComParE feature set $(80.01 \%)$ provides the best UAR, with the 
LDA classifier for emotion recognition. The confusion matrix is shown in Figure 6 for further insight (i.e., precision and recall for all $6+1$ emotions) into the best result. The results indicate that the SVM provides the best averaged UAR of $73.42 \%$ across all the feature sets, and the ComParE feature set $(57.76 \%)$ provides the best average UAR across the all classifiers.

Table 1. Experiment 1: Affect recognition results without identity protection where training and validation is performed on the non-disguised audio data. The Unweighted Average Recall (UAR\%) is reported. The bold figures indicate the highest UARs.

\begin{tabular}{cccccccc}
\hline Features & RF & DT & KNN & NB & SVM & LDA & Avg. \\
\hline emobase. & 0.6835 & 0.5052 & 0.2460 & 0.6051 & 0.7308 & 0.5574 & 0.5547 \\
ComParE & 0.7059 & 0.5368 & 0.2281 & 0.3953 & 0.7949 & $\mathbf{0 . 8 0 0 1}$ & $\mathbf{0 . 5 7 6 8}$ \\
eGeMAPS & 0.7063 & 0.4918 & 0.3885 & 0.4854 & 0.6858 & 0.6616 & 0.5699 \\
\hline avg & 0.6986 & 0.5113 & 0.2875 & 0.4953 & $\mathbf{0 . 7 3 7 2}$ & 0.6730 & - \\
\hline
\end{tabular}

\begin{tabular}{|c|c|c|c|c|c|c|c|c|}
\hline & \multirow{2}{*}{$\begin{array}{l}\text { Recall } \\
90.6 \%\end{array}$} \\
\hline Anger & 115 & & 1 & & 11 & & & \\
\hline Bore. & & 72 & & & & 2 & 7 & $88.9 \%$ \\
\hline Disgust & & & 38 & 3 & 2 & 1 & 2 & $82.6 \%$ \\
\hline Fear & 5 & & 1 & 49 & 9 & 1 & 4 & $71.0 \%$ \\
\hline Happy & 23 & & 2 & 5 & 41 & & & $57.7 \%$ \\
\hline Sad & & 4 & 1 & 2 & & 50 & 5 & $80.6 \%$ \\
\hline Neutral & & 5 & 2 & 1 & & 1 & 70 & $88.6 \%$ \\
\hline Precision & $\begin{array}{l}\text { Anger } \\
80.4 \%\end{array}$ & $\begin{array}{l}\text { Bore. } \\
88.9 \%\end{array}$ & $\begin{array}{r}\text { Disgust } \\
84.4 \%\end{array}$ & $\begin{array}{l}\text { Fear } \\
81.7 \%\end{array}$ & $\begin{array}{l}\text { Happy } \\
65.1 \%\end{array}$ & $\begin{array}{l}\text { Sad } \\
90.9 \%\end{array}$ & $\begin{array}{r}\text { Neutral } \\
79.5 \%\end{array}$ & $\begin{array}{l}\text { UAR }=80.01 \% \\
\text { Accuracy }=81.31 \%\end{array}$ \\
\hline
\end{tabular}

Predicted Class

Figure 6. Confusion matrix of the best result for experiment 1 using LDA and Compare Feature set.

\subsection{Experiment 2}

The UAR for all feature sets and classification methods is shown in Table 2. These results indicate that the combination of the ComParE feature set and LDA again provides the best UAR score $(76.29 \%)$. The confusion matrix for this is shown in Figure 7 where precision and recall for all $6+1$ emotions are listed. In addition, SVM provides the best averaged UAR of $71.68 \%$ across all the feature sets, and the eGeMAPS feature set (54.78\%) provides the best average UAR across the all classifiers.

Table 2. Experiment 2: Affect recognition results with identity protection for training and validation subjects where training and validation are performed on the pitch-shifted audio data. The Unweighted Average Recall (UAR\%) is reported. The bold figures indicate the highest UARs.

\begin{tabular}{cccccccc}
\hline Features & RF & DT & KNN & NB & SVM & LDA & Avg. \\
\hline emobase. & 0.6657 & 0.4588 & 0.2759 & 0.5865 & 0.7358 & 0.5417 & 0.5441 \\
ComParE & 0.7063 & 0.5211 & 0.2016 & 0.2440 & 0.7388 & $\mathbf{0 . 7 6 2 9}$ & 0.5291 \\
eGeMAPS & 0.6335 & 0.4529 & 0.3705 & 0.4818 & 0.6759 & 0.6720 & $\mathbf{0 . 5 4 7 8}$ \\
\hline avg & 0.6685 & 0.4776 & 0.2827 & 0.4374 & $\mathbf{0 . 7 1 6 8}$ & 0.6589 & - \\
\hline
\end{tabular}




\begin{tabular}{|c|c|c|c|c|c|c|c|c|}
\hline \multirow{2}{*}{ Anger } & \multicolumn{8}{|r|}{ Recall } \\
\hline & 103 & & 1 & 4 & 19 & & & $81.1 \%$ \\
\hline Bore. & 1 & 68 & & 1 & 1 & 1 & 9 & $84.0 \%$ \\
\hline $\mathscr{\infty}$ Disgust & 1 & 1 & 35 & 1 & 1 & 1 & 6 & $76.1 \%$ \\
\hline Fear & 9 & & 2 & 48 & 4 & 2 & 4 & $69.6 \%$ \\
\hline$\stackrel{\circlearrowright}{\supset}$ Happy & 26 & & 1 & 6 & 37 & & 1 & $52.1 \%$ \\
\hline Sad & & 1 & 1 & 4 & & 52 & 4 & $83.9 \%$ \\
\hline Neutral & & 6 & 3 & & 1 & & 69 & $87.3 \%$ \\
\hline Precision & $\begin{array}{l}\text { Anger } \\
73.6 \%\end{array}$ & $\begin{array}{r}\text { Bore. } \\
89.5 \%\end{array}$ & $\begin{array}{l}\text { Disgust } \\
81.4 \%\end{array}$ & $\begin{array}{r}\text { Fear } \\
75.0 \%\end{array}$ & $\begin{array}{l}\text { Happy } \\
58.7 \%\end{array}$ & $\begin{array}{r}\text { Sad } \\
92.9 \%\end{array}$ & $\begin{array}{l}\text { Neutral } \\
74.2 \%\end{array}$ & $\begin{array}{l}\text { UAR }=76,29 \% \\
\text { Accuracy }=77.01 \%\end{array}$ \\
\hline
\end{tabular}

Figure 7. Confusion matrix of the best result for experiment 2 using LDA and Compare Feature set.

\subsection{Experiment 3}

The results for this experiment are shown in Table 3. These results indicate that the ComParE feature set again provides the best UAR $(65.13 \%)$, but this time the RF classifier proves to be the most effective. The confusion matrix is shown in Figure 8 where precision and recall for all $6+1$ emotions are listed. RF provides the best averaged UAR of $57.33 \%$ across all feature sets, and the emobase feature set yields the best average UAR across all classifiers $(45.95 \%)$.

Table 3. Experiment 3: Affect recognition results with identity protection for validation subjects, where training is performed on the non-disguised audio data and validation is performed on the pitch-shifted audio data. The Unweighted Average Recall (UAR\%) is reported. The bold figures indicate the highest UARs.

\begin{tabular}{cccccccc}
\hline Features & RF & DT & KNN & NB & SVM & LDA & Avg. \\
\hline emobase. & 0.5624 & 0.4172 & 0.2162 & 0.4838 & 0.6103 & 0.4673 & $\mathbf{0 . 4 5 9 5}$ \\
ComParE & $\mathbf{0 . 6 5 1 3}$ & 0.4479 & 0.2161 & 0.1429 & 0.1435 & 0.1344 & 0.2893 \\
eGeMAPS & 0.5062 & 0.3698 & 0.2623 & 0.3470 & 0.5391 & 0.1339 & 0.3597 \\
\hline avg & $\mathbf{0 . 5 7 3 3}$ & 0.4116 & 0.2315 & 0.3246 & 0.4310 & 0.2452 & - \\
\hline
\end{tabular}

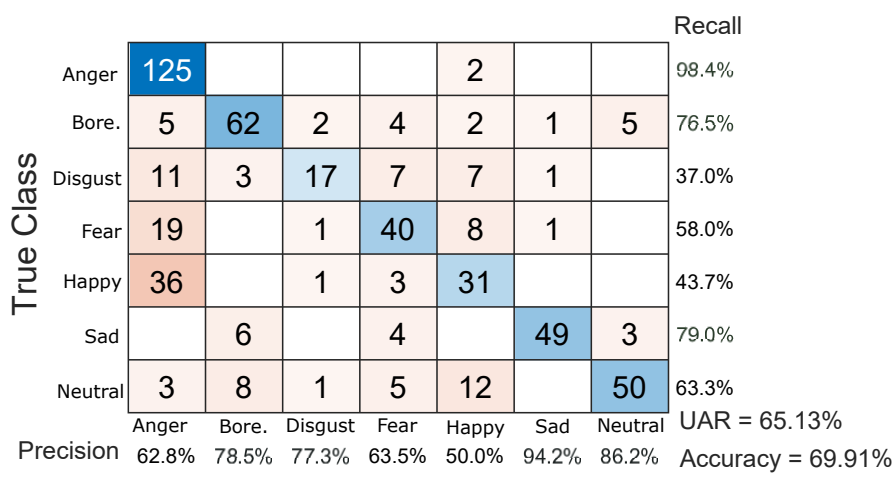

\section{Predicted Class}

Figure 8. Confusion matrix of the best result for experiment 3 using RF and Compare Feature set.

\subsection{Experiment 4}

The resulting UAR scores for all feature sets and classification methods used in this experiment are shown in Table 4. As before, the ComParE/RF combination achieves the best result $(68.32 \%)$. The confusion matrix is shown in Figure 9 where precision and recall for all 6+1 emotions are listed. As in the previous experiment, RF provided the best averaged UAR (60.34\%) across all the feature sets, and the emobase feature set yielded the best average UAR across classifiers (48.62\%). 
Table 4. Experiment 4: Affect recognition results with identity protection, where training and validation are performed on selected acoustic features of the non-disguised audio data and validation is performed on the pitch-shifted audio data. The Unweighted Average Recall (UAR\%) is reported. The bold figures indicate the highest UARs.

\begin{tabular}{cccccccc}
\hline Features & RF & DT & KNN & NB & SVM & LDA & Avg. \\
\hline emobase. & 0.5731 & 0.4121 & 0.2665 & 0.5331 & 0.6250 & 0.5075 & $\mathbf{0 . 4 8 6 2}$ \\
ComParE & $\mathbf{0 . 6 8 3 2}$ & 0.4793 & 0.2541 & 0.1429 & 0.1839 & 0.1231 & 0.3111 \\
eGeMAPS & 0.5540 & 0.4467 & 0.2623 & 0.3305 & 0.4988 & 0.4375 & 0.4216 \\
\hline avg & $\mathbf{0 . 6 0 3 4}$ & 0.4460 & 0.2610 & 0.3355 & 0.4359 & 0.3560 & - \\
\hline
\end{tabular}

\begin{tabular}{|c|c|c|c|c|c|c|c|c|}
\hline & & & & & & & & Recall \\
\hline Anger & 125 & & & 2 & & & & $98.4 \%$ \\
\hline Bore. & 2 & 70 & & 1 & & 1 & 7 & $86.4 \%$ \\
\hline OD Disgust & 8 & 7 & 23 & 7 & & 1 & & $50.0 \%$ \\
\hline Fear & 16 & 1 & 2 & 44 & 3 & 1 & 2 & $63.8 \%$ \\
\hline 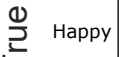 & 44 & 2 & 2 & 5 & 13 & & 5 & $18.3 \%$ \\
\hline $\mathrm{Sad}$ & & 4 & & 4 & & 49 & 5 & $79.0 \%$ \\
\hline Neutral & 1 & 6 & & 6 & 1 & & 65 & $82.3 \%$ \\
\hline & Anger & Bore. & Disgust & Fear & Happy & Sad & Neutral & UAR $=68.32 \%$ \\
\hline Precision & $63.8 \%$ & $77.8 \%$ & $85.2 \%$ & $63.8 \%$ & $76.5 \%$ & $94.2 \%$ & $77.4 \%$ & Accuracy $=72.7$ \\
\hline
\end{tabular}

Figure 9. Confusion matrix of the best result for experiment 4 using RF and Compare Feature set.

\section{Discussion}

The summary of results is shown in Table 5. We note that the non-disguised speech (i.e., Experiment 1) provides the best UAR and accuracy, but experiments 4 and 3 provide the best recall for Anger (98.43\%) and Sad (83.87\%) as shown in bold in Table 5. The 'Happy' emotion is miss-classified as 'Anger' and the miss-classification rate increases for disguised speech experiments, with the worst miss-classification rate occurring when feature selection is performed (Experiment 4). However, feature selection provides better overall UAR (68.32\%) than the full feature set (65.13\%). Experiment 2 provides better UAR $(76.29 \%)$ than experiments 3 and 4 . One of the advantages of the architecture employed in experiment 2 is that the training and testing are both performed on the disguised speech, with the pitch shifted by the same factor (i.e., 2) for all speech utterances. A variable pitch factor may result in a different outcome.

Table 5. Results Summary: Accuracy (Accu.), Unweighted Average Recall (UAR) and recall of each emotion for the best results of each experiment. The bold figures indicate the highest UARs.

\begin{tabular}{cccccccccc}
\hline Experiment & Accu. & UAR & Anger & Bore. & Disgust & Fear & Happy & Sad & Neutral \\
\hline EXP.1 & $\mathbf{8 1 . 3 1}$ & $\mathbf{8 0 . 0 1}$ & 90.55 & $\mathbf{8 8 . 8 9}$ & $\mathbf{8 2 . 6 1}$ & $\mathbf{7 1 . 0 1}$ & $\mathbf{5 7 . 7 5}$ & 80.65 & 88.61 \\
EXP.2 & 77.01 & 76.29 & 81.10 & 83.95 & 76.09 & 69.57 & 52.11 & $\mathbf{8 3 . 8 7}$ & $\mathbf{8 7 . 3 4}$ \\
EXP.3 & 69.91 & 65.13 & $\mathbf{9 8 . 4 3}$ & 76.54 & 36.96 & 57.97 & 43.66 & 79.03 & 63.29 \\
EXP.4 & 72.71 & 68.32 & $\mathbf{9 8 . 4 3}$ & 86.42 & 50.00 & 63.77 & 18.31 & 79.03 & 82.28 \\
\hline
\end{tabular}

To better understand the relationship between the experiments, we also plotted the Venn diagram shown in Figure 10. In this diagram, the brown area (labelled "Target") represents the annotated labels, the blue area represents the predicted labels of Experiment 1, the red area represents the predicted labels of Experiment 2, the green area represents the prediction obtained with the experiment 3 and finally the yellow area represents labels predicted with the experiment 4 . The Venn diagrams suggest the information captured by different pitch profiles is not similar, as only 289 out of 535 instances are detected by all the experiments. 


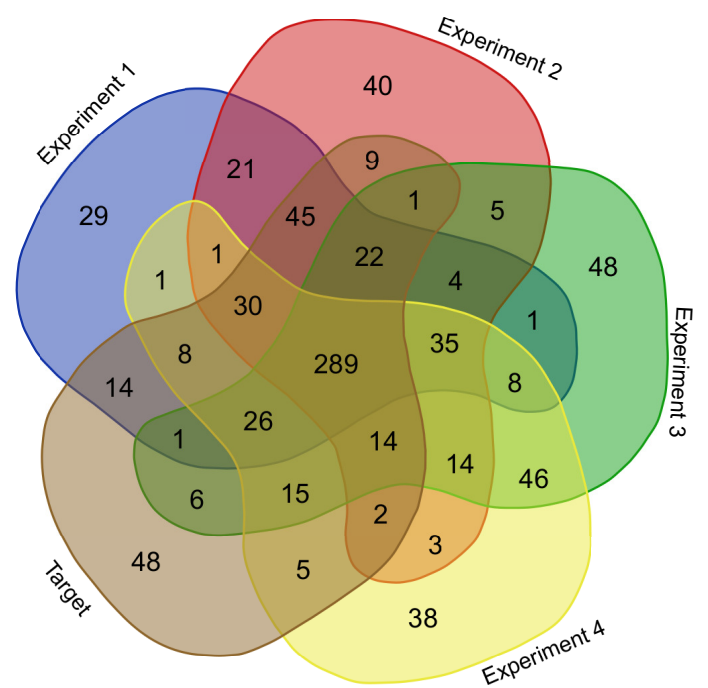

Figure 10. Venn diagram.

While previous studies have proposed affect recognition systems $[3,19,24-26]$, this study presents an analysis of affect recognition on data that have been transformed to protect the identity of users.

\section{Limitations}

Some limitations of this study which we intend to address in future work include:

- the use of an off-the-shelf pitch shifting method which could have an influence on the performance of affect recognition system;

- the fact that pitch is shifted using a constant factor of 2 , whereas a different factor or a variable factor could result in different results;

- feature selection is performed though a statistical approach, and more sophisticated feature selection methods [25] might improve the results further;

- the disguised speech for affect recognition system is evaluated using data which are collected in lab-settings instead of real-world settings;

- the hardware used for the proposed system is a combination of matrix creator and Raspberry Pi $3 \mathrm{~B}+$ with a $1.4 \mathrm{GHz}$ 64-bit quad-core processor.

\section{Conclusions}

AAL can benefit from unobtrusive, privacy-preserving systems for gathering and processing of speech at home. This paper describes a framework for capturing disguised speech and training machine learning models while protecting the identity of users for automatic wellbeing monitoring tasks, in the context of an AAL-based coaching system for healthy ageing. This study also demonstrates that the acoustic information of disguised speech can be used for emotion recognition. We found that, while the non-disguised speech signal gives the best Unweighted Average Recall (UAR) of $80.01 \%$, the disguised speech signal only causes a slight degradation of performance, reaching $76.29 \%$. The transfer learning from non-disguised to disguised speech results in a reduction of UAR $(65.13 \%)$. However, feature selection improves the UAR (68.32\%). Privacy protection and preservation in audio and speech can be regarded from different perspectives, including the protection of a person's identity, protection of the content spoken, and protection from inferences one may be able to draw from the characteristics of a person's voice (such as cognitive or emotional status) [27]. A current limitation of the pitch shifting approach is that it addresses the first (using pitch shifting for identity protection) and second aspects (using statistical functionals of acoustic features instead of content). In the future, we aim to address inference protection within a general framework. We also plan to evaluate humans' annotation performance on disguised speech. 
Author Contributions: Conceptualization, F.H., P.A. and S.L.; Data curation, F.H. and P.A.; Formal analysis, F.H.; Funding acquisition, S.L.; Investigation, F.H., P.A. and S.L.; Methodology, F.H.; Project administration, S.L.; Software, F.H.; Supervision, S.L.; Writing—original draft, F.H.; Writing-review \& editing, F.H., P.A. and S.L. All authors have read and agreed to the published version of the manuscript.

Funding: This research has received funding from the European Union's Horizon 2020 research and innovation programme under Grant No. 769661, SAAM project.

Institutional Review Board Statement: Not applicable.

Informed Consent Statement: Not applicable.

Data Availability Statement: The data can be made availble on request.

Conflicts of Interest: The authors declare no conflict of interest.

\section{References}

1. Dimitrov, Y.; Gospodinova, Z.; Žnidaršič, M.; Ženko, B.; Veleva, V.; Miteva, N. Social Activity Modelling and Multimodal Coaching for Active Aging. In Proceedings of the Personalized Coaching for the Wellbeing of an Ageing Society, $\mathrm{COACH}^{\prime 2019}$, Rhodes, Greece, 5-7 June 2019.

2. Haider, F.; Pollak, S.; Zarogianni, E.; Luz, S. SAAMEAT: Active Feature Transformation and Selection Methods for the Recognition of User Eating Conditions. In Proceedings of the 20th ACM International Conference on Multimodal Interaction, ICMI '18, Boulder, CO, USA, 16-20 October 2018; ACM: New York, NY, USA, 2018; pp. 564-568. [CrossRef]

3. Haider, F.; Luz, S. Attitude recognition using multi-resolution cochleagram features. In Proceedings of the ICASSP 2019-2019 IEEE International Conference on Acoustics, Speech and Signal Processing (ICASSP), Brighton, UK, $12-17$ May 2019; IEEE: Manhattan, NY, USA, 2019; pp. 3737-3741.

4. Luz, S.; la Fuente, S.D. A Method for Analysis of Patient Speech in Dialogue for Dementia Detection. In Proceedings of the Eleventh International Conference on Language Resources and Evaluation (LREC 2018), Miyazaki, Japan, 7-12 May 2018; Kokkinakis, D., Ed.; European Language Resources Association (ELRA): Paris, France, 2018.

5. Hrovat, A.; Znidarsic, M.; Zenko, B.; Vucnik, M.; Mohorcic, M. Saam: Supporting active ageing-use cases and user-side architecture. In Proceedings of the 2018 27th European Conference on Networks and Communications (EuCNC), Ljubljana, Slovenia, 18-21 June 2018.

6. Bondi, M.W.; Salmon, D.P.; Kaszniak, A.W. The neuropsychology of dementia. In Neuropsychological Assessment of Neuropsychiatric Disorders, 2nd ed.; Oxford University Press: New York, NY, USA, 1996; pp. 164-199.

7. Hart, R.P.; Kwentus, J.A.; Taylor, J.R.; Harkins, S.W. Rate of forgetting in dementia and depression. J. Consult. Clin. Psychol. 1987, 55, 101-105. [CrossRef] [PubMed]

8. Lopes, P.N.; Brackett, M.A.; Nezlek, J.B.; Schütz, A.; Sellin, I.; Salovey, P. Emotional intelligence and social interaction. Personal. Soc. Psychol. Bull. 2004, 30, 1018-1034. [CrossRef]

9. Haider, F.; De La Fuente Garcia, S.; Albert, P.; Luz, S. Affective Speech for Alzheimer's Dementia Recognition. In Proceedings of the LREC: Resources and ProcessIng of Linguistic, Para-Linguistic and Extra-Linguistic Data from People with Various Forms of Cognitive/Psychiatric/Developmental Impairments (RaPID), Marseille, France, 11 May 2020; Kokkinakis, D., Lundholm Fors, K., Themistocleous, C., Antonsson, M., Eckerström, M., Eds.; European Language Resources Association (ELRA): Paris, France, 2020; pp. 67-73.

10. Burkhardt, F.; Paeschke, A.; Rolfes, M.; Sendlmeier, W.F.; Weiss, B. A database of German emotional speech. In Proceedings of the Ninth European Conference on Speech Communication and Technology, Lisbon, Portugal, 4-8 September 2005; pp. 1516-1520.

11. Becker, J.; Boller, F.; Lopez, O.; Saxton, J.; McGonigle, K. The natural history of Alzheimer's disease: Description of study cohort and accuracy of diagnosis. Arch. Neurol. 1994, 51, 585-594. [CrossRef]

12. Parliament and the Council. Regulation (EU) 2016/679 on the Protection of Natural Persons with Regard to the Processing of Personal Data and on the Free Movement of Such Data, and Repealing Directive 95/46/EC (General Data Protection Regulation). 2016. Available online: https:/ / eur-lex.europa.eu/legal-content/EN/TXT/?uri=CELEX\%3A02016R0679-20160504 (accessed on 23 November 2021).

13. Nautsch, A.; Jiménez, A.; Treiber, A.; Kolberg, J.; Jasserand, C.; Kindt, E.; Delgado, H.; Todisco, M.; Hmani, M.A.; Mtibaa, A.; et al. Preserving privacy in speaker and speech characterisation. Comput. Speech Lang. 2019, 58, 441-480. [CrossRef]

14. Dimitrievski, A.; Zdravevski, E.; Lameski, P.; Trajkovik, V. Addressing Privacy and Security in Connected Health with Fog Computing. In Proceedings of the 5th EAI International Conference on Smart Objects and Technologies for Social Good. Association for Computing Machinery, GoodTechs '19, Valencia, Spain, 25-27 September 2019; pp. 255-260. [CrossRef]

15. Haider, F.; Luz, S. A System for Real-Time Privacy Preserving Data Collection for Ambient Assisted Living. In Proceedings of the INTERSPEECH, Graz, Austria, 15-19 September 2019; pp. 2374-2375.

16. Perrot, P.; Aversano, G.; Chollet, G. Voice disguise and automatic detection: review and perspectives. In Progress in Nonlinear Speech Processing; Springer: Berlin/Heidelberg, Germany, 2007; pp. 101-117. 
17. Zheng, L.; Li, J.; Sun, M.; Zhang, X.; Zheng, T.F. When Automatic Voice Disguise Meets Automatic Speaker Verification. IEEE Trans. Inf. Forensics Secur. 2020, 16, 824-837. [CrossRef]

18. Haider, F.; Luz, S. Affect Recognition Through Scalogram and Multi-Resolution Cochleagram Features. In Proceedings of the Interspeech 2021, Brno, Czechia, 30 August-3 September 2021; pp. 4478-4482. [CrossRef]

19. Eyben, F.; Scherer, K.R.; Schuller, B.W.; Sundberg, J.; André, E.; Busso, C.; Devillers, L.Y.; Epps, J.; Laukka, P.; Narayanan, S.S.; et al. The Geneva minimalistic acoustic parameter set (GeMAPS) for voice research and affective computing. IEEE Trans. Affect. Comput. 2016, 7, 190-202. [CrossRef]

20. Lajmi, L. An Improved Packet Loss Recovery of Audio Signals Based on Frequency Tracking. J. Audio Eng. Soc. 2018, 66, 680-689. [CrossRef]

21. Boersma, P.; Weenink, D. Praat: Doing Phonetics by Computer [Computer Program]; Version 6.0. 37. 2018; Volume 14, p. 2018. Available online: http:/ / www.praat.org/ (accessed on 1 March 2021).

22. Eyben, F.; Weninger, F.; Groß, F.; Schuller, B. Recent developments in opensmile, the munich open-source multimedia feature extractor. In Proceedings of the 21st ACM International Conference on Multimedia, Barcelona, Spain, 21-25 October 2013; ACM, Association for Computing Machinery: New York, NY, USA, 2013; pp. 835-838.

23. Eyben, F.; Wöllmer, M.; Schuller, B. Opensmile: the munich versatile and fast open-source audio feature extractor. In Proceedings of the 18th ACM international conference on Multimedia, Firenze, Italy, 25-29 October 2010; ACM, Association for Computing Machinery: New York, NY, USA, 2010; pp. 1459-1462.

24. Haider, F.; Salim, F.A.; Conlan, O.; Luz, S. An Active Feature Transformation Method for Attitude Recognition of Video Bloggers. In Proceedings of the INTERSPEECH, Hyderabad, India, 2-6 September 2018; pp. 431-435.

25. Haider, F.; Pollak, S.; Albert, P.; Luz, S. Emotion recognition in low-resource settings: An evaluation of automatic feature selection methods. Comput. Speech Lang. 2020, 65, 101119. [CrossRef]

26. Haider, F.; Pollak, S.; Albert, P.; Luz, S. Extracting Audio-Visual Features for Emotion Recognition Through Active Feature Selection. In Proceedings of the 2019 IEEE Global Conference on Signal and Information Processing (GlobalSIP), Ottawa, ON, Canada, 11-14 November 2019; IEEE: New York, NY, USA, 2019; pp. 1-5.

27. Pathak, M.A.; Raj, B.; Rane, S.D.; Smaragdis, P. Privacy-preserving speech processing: cryptographic and string-matching frameworks show promise. IEEE Signal Process. Mag. 2013, 30, 62-74. [CrossRef] 